Georgian Mathematical Journal

Volume 8 (2001), Number 4, 785-790

\title{
ON THE PRODUCT OF SEPARABLE METRIC SPACES
}

\author{
D. KIGHURADZE
}

\begin{abstract}
Some properties of the dimension function dim on the class of separable metric spaces are studied by means of geometric construction which can be realized in Euclidean spaces. In particular, we prove that if $\operatorname{dim}(X \times$ $Y)=\operatorname{dim} X+\operatorname{dim} Y$ for separable metric spaces $X$ and $Y$, then there exists a pair of maps $f: X \rightarrow \mathbb{R}^{s}, g: Y \rightarrow \mathbb{R}^{s}, s=\operatorname{dim} X+\operatorname{dim} Y$, with stable intersections.
\end{abstract}

2000 Mathematics Subject Classification: 54F45.

Key words and phrases: Essential map, irrational set, stable intersection.

\section{INTRODUCTION}

In this paper I try to answer the question which was posed by Chogoshvili [2]: Is it true that a subset $X$ of $n$-dimensional Euclidean space $\mathbb{R}^{n}$ is at most $k$-dimensional $(-1 \leq k \leq n)$ if and only if for every $(n-k-1)$-dimensional plane $L \subset \mathbb{R}^{n}$ there exists an $\varepsilon$-translation $f: X \rightarrow \mathbb{R}^{n}$ with $f(X) \cap L=\varnothing$ ?

At first Sitnikov proved that this is not true for arbitrary subspaces of Euclidean spaces (see [1]) and then Dranishnikov [3] showed that this is false even for compact subspaces.

It turns out that the answer is positive for the class of irrational subspaces of Euclidean spaces (see Section 2), which is an easy consequence of the following

Theorem 1. Let $X \subset \mathbb{R}^{n}, \operatorname{dim} X=\operatorname{dim} \bar{X}=k(k \leq n)$, and $\bar{X}$ be a $k$ dimensional irrational compact. Then there exist a $k$-dimensional plane $L \subset \mathbb{R}^{n}$ and a closed, $k$-dimensional ball $C^{k} \subseteq L$, such that $\left.P\right|_{\left(P^{-1}\left(C^{k}\right) \cap X\right)}: P^{-1}\left(C^{k}\right) \cap$ $X \rightarrow C^{k}$ is an essential map, where $P: \mathbb{R}^{n} \rightarrow L$ is the orthogonal projection of $\mathbb{R}^{n}$ onto L.

This leads us to the characterization of the dimension of the Cartesian product of separable metric spaces in terms of essential maps (see Section 2).

Theorem 2. For every pair of separable metric spaces $X$ and $Y$, where $\operatorname{dim} X=n, \operatorname{dim} Y=m$, we have $\operatorname{dim}(X \times Y)=n+m$ if and only if there exist essential maps $f: X \rightarrow C^{n}$ and $g: Y \rightarrow C^{m}$ such that $f \times g: X \times Y \rightarrow C^{n+m}$ is essential too.

In 1991 Dranishnikov, Repovš and Ščepin [4] proved that given compacts $X$ and $Y$ in $\mathbb{R}^{n}$ such that $n=\operatorname{dim} X+\operatorname{dim} Y$, there exists a pair of maps $f: X \rightarrow \mathbb{R}^{n}, g: Y \rightarrow \mathbb{R}^{n}$ with stable intersections if and only if $\operatorname{dim}(X \times$ 
$Y)=n$. The proof of the "if" part of this theorem was based on my earlier result, where I considered irrational compact subspaces instead of arbitrary irrational subspaces as in Theorem 1. The corresponding general assertion based on Theorem 1 can be formulated as

Corollary. For every pair of separable metric spaces $X$ and $Y$, where $\operatorname{dim} X=n, \operatorname{dim} Y=m$, and $\operatorname{dim}(X \times Y)=n+m$, there exists a pair of maps $f: X \rightarrow \mathbb{R}^{n+m}$ and $g: Y \rightarrow \mathbb{R}^{n+m}$ with stable intersection.

\section{Notions, Definitions and Auxiliary Theorems}

We denote by dim and $\mu$ dim covering and metric dimension functions, respectively, by $C\left(X, \mathbb{R}^{n}\right)$ the space of all continuous maps of $X$ into $\mathbb{R}^{n}$, equipped by the standard metric: $\rho(f, g)=\sup \{d(f(x), g(x)) \mid x \in X\}$ and denote by $E\left(X, \mathbb{R}^{n}\right)$ the subspace of $C\left(X, \mathbb{R}^{n}\right)$, consisting of all embedings of $X$ into $\mathbb{R}^{n}$. For $X \subset \mathbb{R}^{n}$, we denote by $\bar{X}$ the closure of $X$ into $\mathbb{R}^{n}$ and by $\partial X$ the boundary $X$ in $\mathbb{R}^{n}$.

A map $f: X \rightarrow Q^{n}$ of a space $X$ onto the closed $n$-cube is said to be essential if there is no map $g: X \rightarrow \partial Q^{n}$ with the property that $\left.g\right|_{f^{-1}\left(\partial Q^{n}\right)}=\left.f\right|_{f^{-1}\left(\partial Q^{n}\right)}$. Next a point $x \in \operatorname{int} Q^{n}$ is called a stable value of a surjective map $f: X \rightarrow Q^{n}$ if there exists $\varepsilon>0$ such that for every map $g: X \rightarrow Q^{n}$ such that $\rho(f, g)<\varepsilon$ it follows that $x \in g(X)([1])$. Clearly, if $f: X \rightarrow Q^{n}$ is an essential map, then every point $x \in \operatorname{int} Q^{n}$ is stable value of $f$. Conversely, if some point $x \in Q^{n}$ is a stable value of an onto map $f: X \rightarrow Q^{n}$, then there exists a small $n$-ball $C^{n} \subset \operatorname{int} Q^{n}$ such that $x \in \operatorname{int} C^{n}$ and $\left.f\right|_{f^{-1}\left(C^{n}\right)}: f^{-1}\left(C^{n}\right) \rightarrow C^{n}$ is essential.

Two maps $f: X \rightarrow \mathbb{R}^{n}$ and $g: Y \rightarrow \mathbb{R}^{n}$ have a stable interestion in $\mathbb{R}^{n}$ if there exists $\varepsilon>0$ such that for any $\varepsilon$-permutations $f^{\prime}$ and $g^{\prime}$ of $f$ and $g$, respectively, we have $f^{\prime}(X) \cap g^{\prime}(Y)=\varnothing$.

For every point $x \in \mathbb{R}^{n}$ let $r(x)$ be the number of rational coordinates of $x$. For every subset $X \subset \mathbb{R}^{n}$ let $r(X)=\max \{r(x): x \in X\}$. A subset $X \subset \mathbb{R}^{n}$ is said to be irrational if $r(X)=\operatorname{dim} X$. Finally, for every $k \leq n$, let $R_{k}^{n}=\left\{x \in \mathbb{R}^{n}: r(x) \leq k\right\}$.

We shall need the following classical results of dimension theory (see [1]):

Nobeling-Hurewicz theorem. Every bounded map $f: X \rightarrow \mathbb{R}^{2 n+1}$ of a separable metric $n$-dimensional space $X$ into $\mathbb{R}^{2 n+1}$ can be approximated arbitrarily closely by a map $g: X \rightarrow \mathbb{R}^{2 n+1}$ such that the closure of the image of $g$ is an irrational $n$-dimensional compact.

Sitnikov theorem. If $X \subset \mathbb{R}^{n}$ and $\operatorname{dim} X=\operatorname{dim} \bar{X}$, then $\mu \operatorname{dim} X=$ $\operatorname{dim} \bar{X}$.

Borsuk theorem. If mapping $f: X \rightarrow Q^{k}$ from normal space $X$ onto $k$ dimensional cube is essential, then for every face $Q^{p}$ of $Q^{k}(p \leq k)$, the mapping $\left.f\right|_{f^{-1}\left(Q^{p}\right)}: f^{-1}\left(Q^{p}\right) \rightarrow Q^{p}$ is essential too. 


\section{Proofs}

We shall need two lemmas:

Lemma 1. Given $X \subset \mathbb{R}^{n}$, let $P: \mathbb{R}^{n} \rightarrow \mathbb{R}^{n}, n \geq m$, be a surjective linear map such that $\left.P\right|_{X}: X \rightarrow \mathbb{R}^{m}$ has an unstable value $y \in P(X)$. Then for every $\varepsilon>0$ there exists a map $g: X \rightarrow \mathbb{R}^{n}$ such that :

(a) $\rho(g, j)<\varepsilon$, where $j: X \rightarrow \mathbb{R}^{n}$ is the inclusion;

(b) if $x \in X$ and $\operatorname{dist}\left(x, P^{-1}(y)\right) \geq \varepsilon / 2$, then $g(x)=x$;

(c) $g(X) \cap P^{-1}(y)=\varnothing$.

Proof. Without loss of generality, we may assume that the point $y$ is the origin $O \in \mathbb{R}^{n}$ and that $P$ is the projection of $\mathbb{R}^{n}$ onto the first $m$ coordinates, i.e., $p\left(x_{1}, \ldots, x_{m}, \ldots, x_{n}\right)=\left(x_{1}, \ldots, x_{m}\right)$. Since $y$ is by hypothesis an unstable value of $\left.P\right|_{X}: X \rightarrow \mathbb{R}^{m}$, the map $\left.P\right|_{X}$ unessentially covers the closed $m$-ball $C^{m} \subset \mathbb{R}^{m}$, centered at $y$ and with radius $\varepsilon / 2$. Hence there exists a map $f: X \rightarrow \mathbb{R}^{m}$ such that $f\left(X \cap P^{-1}\left(C^{m}\right)\right) \subset \partial C^{m}$ and for every $x \in X \cap \overline{P^{-1}\left(\mathbb{R}^{m} \backslash C^{m}\right)}, f(x)=P(x)$. Define now the desired map $g: X \rightarrow \mathbb{R}^{n}$ by $g(x)=\left(f_{1}(x), \ldots, f_{m}(x), x_{m+1}, \ldots, x_{n}\right)$ for every $x \in X$, where $x=\left(x_{1}, \ldots, x_{n}\right)$ and $f(x)=\left(f_{1}(x), \ldots, f_{m}(x)\right)$. It is now easy to verify that $g$ satisfies properties (a), (b), (c).

Lemma 2. Suppose that a compact $X \subset \mathbb{R}^{n}$ and a collection $L_{1}, \ldots, L_{k} \subset$ $\mathbb{R}^{n}$ of planes satisfy the following conditions:

(1) for every $i \neq j, X \cap L_{i} \cap L_{j}=\varnothing$;

(2) for every $i$, the projection $\left.P_{L_{i}}\right|_{X}: X \rightarrow L_{i}$ has an unstable point at $P_{L_{i}}\left(L_{i}\right)$.

Then for every $\varepsilon>0$ there exists a map $g: X \rightarrow \mathbb{R}^{n}$ such that:

(a) $\rho(g, j)<\varepsilon$, where $j ; X \rightarrow \mathbb{R}^{n}$ is the inclusion;

(b) $g(X) \cap\left(\cup_{i=1}^{k} L_{i}\right)=\varnothing$.

Proof. We may assume that $\varepsilon>0$ is so small that for every $i \neq j$,

$$
X \cap N_{\varepsilon}\left(L_{j}\right) \cap N_{\varepsilon}\left(L_{j}\right)=\varnothing,
$$

where $N_{\varepsilon}\left(L_{j}\right) \subset \mathbb{R}^{n}$ is the open $\varepsilon$-neighborhood of $L_{i}, i \in\{1, \ldots, k\}$. Apply Lemma 1 to obtain, for every $i \in\{1, \ldots, k\}$, a map $g: X \rightarrow \mathbb{R}^{n}$ such that

$$
\begin{gathered}
\rho\left(g_{i}, \text { incl }\right)<\varepsilon, \quad \text { for every } \quad x \in X, \\
g_{i}(x)=x, \quad \text { if } \operatorname{dist}\left(x, L_{i}\right) \geq \varepsilon \text { and } \\
g_{i}(X) \cap L_{i}=\varnothing
\end{gathered}
$$

(here "incl" denotes the inclusion map).

Define $g: X \rightarrow \mathbb{R}^{n}$ as follows: for every $x \in X$. let $g(x)=g_{i}(x)$, where $L_{i}$ is the closest plane to $x$, i.e., $\operatorname{dist}\left(x, L_{i}\right) \leq \operatorname{dist}\left(x, L_{j}\right), j \in\{1, \ldots, k\}$. The map $g$ is well defined. Indeed, if for some $i \neq j$, the planes $L_{i}$ and $L_{j}$ both have a minimal distance from $x$, then by (1) above this distance must be at least $\varepsilon$. Consequently, by $(3), g_{i}(x)=x=g_{j}(x)$. It is clear, by $(1)-(4)$, that $g$ is continuous and, as follows from (1)-(4), it satisfies the required conditions. 
Proof of Theorem 1 is given here for completeness although it is similar to the case when $X$ is compact. Let $X \subset \mathbb{R}^{n}$ and $\bar{X}$ be a $k$-dimensional irrational compact and $\operatorname{dim} X=\operatorname{dim} \bar{X}$. By Sitnikov's theorem we have $\mu \operatorname{dim} X=k$. Then:

(i) for every $x \in X, r(x) \leq k$;

(ii) there exists $\delta>0$ such that $X$ has no open $\delta$-covering of order $\leq k$.

For a retional number $\lambda$ such that $0<\lambda<\delta \sqrt{n} / 2$ define $C_{\lambda}=\left\{x \in \mathbb{R}^{n}\right.$, $0 \leq x_{i} \leq \lambda / n$, for every $\left.i\right\}$. We have $d=\operatorname{diam} C_{\lambda}=\lambda / \sqrt{n}<\delta / 2$.

Consider a Lebesgue lattice $\Omega=\left\{w_{i}\right\}_{i \in N}=$ in $\mathbb{R}^{n}$, i.e., the covering of $\mathbb{R}^{n}$ by copies of the $n$-cube $C_{\lambda}$ such that:

(a) for every $i, w_{i}=C_{\lambda}+\bar{r}_{i}, \bar{r}_{i} \in \mathbb{Q}^{n}$ (which is the $n$th Cartesian power of the set $\mathbb{Q}$ of all rational numbers), i.e., $w_{i}$ is obtained by a parallel translation of $C_{\lambda}$ along some rational vector $r_{i}$;

(b) for every $i \neq j=, w_{i} \cap w_{j}=\partial w_{i} \cap w_{j}$;

(c) the order of $\Omega$ is $n+1$.

For every $m \geq 1$, define

$$
S_{m}=\left\{x \in \mathbb{R}^{n} \mid x \text { belongs to at least } m \text { different elements of } \Omega\right\} .
$$

Then

$$
S_{m} \subset \cap_{j=1}^{\infty} L_{j}^{n-m+1}
$$

where $\left\{L_{j}^{n-m+1}\right\}_{j \in N}$ is a discrete collection of $(n-m+1)$-dimensional planes in $\mathbb{R}^{n}$, each of them being the intersection of some $m-1$ hyperplanes $\left\{\Sigma_{\ell}^{n-1} \mid \ell=\right.$ $1, \ldots, m-1\}$,

$$
L_{j}^{n-m+1}=\bigcap_{e=1}^{m-1} \Sigma_{\ell}^{n-1},
$$

where for every $\ell \in\{1, \ldots, m-1\}$ and for some $t(\ell) \in\{1, \ldots, n\}$ and $q(\ell) \in \mathbb{Q}$

$$
\Sigma_{\ell}^{n-1}=\left\{\left(x_{i}, \ldots, x_{n}\right) \in \mathbb{R}^{n} \mid x_{t(\ell)}=q(\ell)\right\} .
$$

We now focus our attention on the case $m=k+1$. Note that for every $i \in N$ and every $y \in L_{i}^{n-k}, r(y) \geq k$, hence for every $i \neq j$ and every $z \in L_{i}^{n-k} \cap L_{j}^{n-k}$, $r(z) \geq k+1$, therefore it follows from (1) that for every $i \neq j$,

$$
X \cap L_{i}^{n-k} \cap L_{j}^{n-k}=\varnothing .
$$

Let $\varepsilon_{0}=(\delta-2 d) / 2$ which is positive since $d<\delta / 2$. It follows from (4) that $\varepsilon_{0}>0$.

Proposition. For every map $g: X \rightarrow \mathbb{R}^{n}$ such that $\rho(g$, incl $)<\varepsilon_{0}$ we have $g(X) \cap S_{k+1} \neq \varnothing$.

To prove this proposition suppose, on the contrary, that the intersection of $g(X)$ and $S_{k+1}$ is empty. Then $g^{-1}\left(\Omega^{\prime}\right)$ will provide an open cover of $X$ of order $\leq k$ and with mesh $\mu<2 \varepsilon_{0}+2 d=\delta$, where $\Omega^{\prime}=\left\{w_{i}^{\prime}\right\}_{i \in N}$ is some family of open cubs $w_{i}^{\prime} \supset w_{i}$ which directly contradicts (2). This proves the proposition. 
The proposition implies, in particular, that

$$
X \cap S_{k+1} \neq \varnothing
$$

and since $\bar{X}$ is compact, it intersects only finitely many $(n-k)$-dimensional planes $\left\{L_{j}^{n-k}\right\}_{j \in N}$, say $L_{\sigma(1)}^{n-k}, \ldots, L_{\sigma(l)}^{n-k}$. By discreteness, there is $\left.\varepsilon_{1} \in\right] 0, \varepsilon_{0}[$ such that $\rho(g$, incl $) \leq \varepsilon_{1}$ Proposition implies, that

$$
g(X) \cap\left(\cup_{i=l}^{l} L_{\sigma(i)}^{n-k}\right)=\varnothing .
$$

It follows now from (7), (9) and Lemma 2, that for some $i_{0} \in\{1, \ldots, l\}$, the projection $P_{L_{\sigma\left(i_{0}\right)}} \mid X: X \rightarrow L_{\sigma\left(i_{0}\right)}^{\perp}$ has a stable value at the point $P_{L \sigma\left(i_{0}\right)}\left(L_{\sigma\left(i_{0}\right)}\right)$. Now let $L=L_{\sigma\left(i_{0}\right)}^{\perp}$ and $P=P_{L_{\sigma\left(i_{0}\right)}}$. Thus Theorem 1 is proved.

Remark. It is evident from the proof of Theorem 1 that $L_{\sigma\left(i_{0}\right)}$ and $L_{\sigma\left(i_{0}\right)}^{\perp}$ are $(n-k)$ - and $k$-dimensional coordinate planes, respectively.

Proof of Theorem 2. The "if" part of Theorem 2 is clear. Let $i: X \rightarrow \mathbb{R}^{2 n+1}$ and $j: Y \rightarrow \mathbb{R}^{2 m+1}$ be embeddings of $X$ and $Y$, respectively, such that $\operatorname{dim} \overline{i(X)}=$ $\operatorname{dim} X=n, \operatorname{dim} \overline{j(Y)}=\operatorname{dim} Y=m$, and $\overline{i(X)}$ and $\overline{j(Y)}$ are irrational compacts (we apply the Nőbeling-Hurewicz theorem).

Consider the product of these embeddings $i \times j: X \times Y \rightarrow \mathbb{R}^{2 n+2 m+2}$. It is easy to see that $\overline{(i \times j)(X \times Y)}=\overline{i(X)} \times \overline{j(Y)}$ is an irrational compact. Hence by Theorem 1 and Remark there exist an $(n+m)$-dimensional plane $L \subset \mathbb{R}^{2 n+2 m+2}$ and a closed $(n+m)$-dimensional cube $Q^{n+m} \subset L$ such that $\left.P\right|_{P^{-1}\left(Q^{n+m}\right) \cap(i \times j)(X \times Y)}: P^{-1}\left(Q^{n+m}\right) \cap(i \times j)(X \times Y) \rightarrow Q^{n+m}$ is an essential map.

Assume that all points $z$ from $L$ have $p$ " $x$ "-coordinates and $q$ " $y$ "-coordinates: $z=\left(x_{i_{1}}, \ldots, x_{i_{p}}, y_{j_{1}}, \ldots, t_{j_{q}}\right)$, where $1 \leq j_{1}<\cdots<j_{q} \leq 2 m+1$ and $1 \leq j_{1}<$ $\cdots<j_{q} \leq 2 n+1$, such that $p+q=n+m$.

Let $\pi_{x}: Q^{n+m} \rightarrow Q_{x}^{p}$ and $\pi_{y}: Q^{n+m} \rightarrow Q_{y}^{q}$ be projections of $Q^{n+m}$ an its $p$ and $q$-dimensional " $X$ " and " $Y$ " faces.

The Borsuk theorem implies that the maps

$$
\begin{aligned}
& \left.\pi_{x} \circ P\right|_{P^{-1}\left(Q^{n+m}\right) \cap(i \times j)(X \times Y)}: P^{-1}\left(Q^{n+m}\right) \cap(i \times j)(X \times Y) \rightarrow Q_{x}^{p} L^{p}, \\
& \left.\pi_{y} \circ P\right|_{P^{-1}\left(Q^{n+m}\right) \cap(i \times j)(X \times Y)}: P^{-1}\left(Q^{n+m}\right) \cap(i \times j)(X \times Y) \rightarrow Q_{y}^{q} L^{q}
\end{aligned}
$$

are essential and therefore $p=n, q=m$, because if we assume the contrary, we obtain $p>n$ or $q>m$, which is impossible, so $Q_{x}^{p} \equiv Q_{x}^{n}$ and $Q_{y}^{q} \equiv Q_{y}^{m}$.

Let $c^{\prime}$ and $c^{\prime \prime}$ be the centers of $Q_{x}^{n}$ and $Q_{y}^{m}$, respectively. Define the maps $f: X \rightarrow Q_{x}^{n}$ and $g: Y \rightarrow Q_{y}^{m}$ as follows:

$$
\begin{aligned}
& f(x)= \begin{cases}i(x), & i(x) \in Q_{x}^{n}, \\
{\left[c^{\prime}, j(x)\right] \cap \partial Q_{x}^{n},} & i(x) \in Q_{x}^{n},\end{cases} \\
& g(y)= \begin{cases}j(y), & j(y) \in Q_{y}^{m}, \\
{\left[c^{\prime \prime}, j(y)\right] \cap \partial Q_{y}^{m},} & j(y) \in Q_{y}^{m} .\end{cases}
\end{aligned}
$$


It is easy to see that $f$ and $g$ are the required maps.

Proof of Corollary. (This proof proceeds according to the author's one from [4].) By Theorem 2 there exist such maps $f: X \rightarrow Q^{n} \subset \mathbb{R}^{n}$ and $g: Y \rightarrow Q^{m} \subset \mathbb{R}^{m}$, that $f \times g: X \times Y \rightarrow Q^{n} \times Q^{m} \subset \mathbb{R}^{n+m}$ is essential. Without loss of generality, we can assume that $f(X) \cap f(Y)=O=c^{\prime} \times c^{\prime \prime} \in Q^{n} \times Q^{m}$, where $c^{\prime}$ and $c^{\prime \prime}$ are centers of $Q^{\prime \prime}$ and $Q^{\prime \prime \prime}$ and $O$ is the origin of Euclidean space $\mathbb{R}^{n+m}$.

The maps $f$ and $g$ have a stable intersection.

Indeed, it is clear that the map $f \times(-g): X \times Y \rightarrow Q^{n+m}$, where $(f \times$ $(-g))(x, y)=(x,-y)=f(x)-g(y)$ (here we consider $f(x)$ and $g(y)$ as vectors in the Euclidean space $\left.\mathbb{R}^{n+m}\right)$, is essential. Hence we obtain that there exists $\varepsilon>0$ such that for every map $H: X \times Y \rightarrow Q^{n+m}$, with $\left.\rho(f \times(-g)), H\right)<\varepsilon$, we have $O \in H(X \times Y)$.

Suppose that $f$ and $g$ have not a stable intersection. Then there exist $f^{\prime}$ : $X \rightarrow \mathbb{R}^{n+m}$ and $g^{\prime}: Y \rightarrow \mathbb{R}^{n+m}$, satisfying the conditions: $\rho\left(f, f^{\prime}\right)<\varepsilon / 4$, $\rho\left(g, g^{\prime}\right)<\varepsilon / 4$ and $f^{\prime}(X) \cap g^{\prime}(Y)=\varnothing$. Define $H: X \times Y \rightarrow \mathbb{R}^{n+m}$ as $H(x, y)=$ $f^{\prime}(x)-g^{\prime}(y)$. Then $O \notin H(X \times Y)$. On the other hand, $\rho(f \times(-g), H) \leq$ $\rho\left(f, f^{\prime}\right)+\rho\left(g, g^{\prime}\right)<\varepsilon / 2$. This is a contradiction.

\section{REFERENCES}

1. P. S. Aleksandrov and B. A. Pasynkov, Introduction to dimension theory. (Russian) Nauka, Moscow, 1973.

2. G. Chogoshvili, On a theorem in the theory of dimensionality. Compositio Math. 5, No. 2(1937), 292-298.

3. A. N. Dranishnikov, On Chogoshvili's conjecture. Proc. Amer. Math. Soc. 125(1997), No. 7, 2155-2160.

4. A. N. Dranišnikov, D. Repovš, and E. V. Ščepin, On intersections of compacta of complementary dimensions in Euclidean space. Topology Appl. 38(1991), 237-253.

(Received 5.11.1999; revised 10.09.2001)

Author's address:

Faculty of Mechanics and Mathematics

I. Javakhishvili Tbilisi State University

2, University St., Tbilisi 380043

Georgia

Current address:

Oklahoma State University

Department of Mathematics

401 Math. Science Building

Stillwater, OK 74078

U.S.A.

E-mail: d_kighuradze@hotmail.com 